Background Central venous catheter (CVC) associated sepsis is a common complication in children with intestinal failure on longterm parenteral nutrition, and is associated with significant morbidity and mortality.

We aimed to examine whether a strategy of using daily prophylactic antibiotic line locks (Gentamicin, Vancomycin or Amikacin) in children with intestinal failure on long-term parenteral nutrition, at high risk of CVC-associated sepsis, reduced rates of CVC-associated sepsis and antibiotic usage.

Methods Retrospective review of clinical notes of 12 children in whom antibiotic line locks have been used in the preceding five years, analysing rates of suspected and confirmed (blood culture or bacterial DNA positive) CVC-associated sepsis and in-patient antibiotic days, off and on antibiotic line locks using each child as their own historical control. Data was analysed using STATA version 10, using Cox proportional hazards survival models.

Results The twelve children studied received parenteral nutrition for a total of 8003 CVC days over the study period (5709 off lock, and 2294 on antibiotic line lock.) There were 99 episodes of suspected CVC-associated sepsis, and 43 episodes of confirmed (blood-culture/bacterial DNA positive) CVC-associated sepsis.

Children on antibiotic line locks had reduced rates of confirmed CVC-associated sepsis from 6.1 to 3.5 episodes per 1000 CVC days but this was not statistically significant $(p=0.2$.) They also had reduced rates of suspected CVC-associated sepsis from 14.7 to 8.7 episodes per $1000 \mathrm{CVC}$ days $(\mathrm{p}=0.04$.) Mean rates of in-patient antibiotic days were reduced on antibiotic locks; 148 compared with 89 days/per 1000 CVC days, $\mathrm{p}=0.8$.

Younger children were at higher risk of having an episode of suspected CVC associated sepsis than older children. Children under two years of age had significantly higher rates of gramnegative sepsis than children over two years of age $(p=0.034$.) Given the high mortality associated with gram-negative sepsis this group may benefit the most from prophylactic antibiotic line locks

Conclusion Antibiotic line locks may reduce rates of CVC-associated sepsis and in-patient antibiotic days in children with intestinal failure on long-term parenteral nutrition. In view of the potential implications of these findings, further larger randomised studies are required to evaluate this approach.

\section{G366(P) IMPEDANCE MONITORING IN PAEDIATRIC GASTRO- OESOPHAGEAL REFLUX DISEASE: AN EVALUATION OF ITS USE AND CLINICAL VALUE}

${ }^{1} \mathrm{P}$ Crook, ${ }^{2} \mathrm{~A}$ Ross, ${ }^{3} \mathrm{R}$ Heuschkel, ${ }^{2,3} \mathrm{M}$ Zilbauer. ${ }^{1}$ School of Clinical Medicine, University of Cambridge, Cambridge, UK; ${ }^{2}$ Department of Paediatrics, University of Cambridge, Cambridge, UK; ${ }^{3}$ Department of Paediatric Gastroenterology, Hepatology and Nutrition, Addenbrooke's Hospital, Cambridge, UK

\subsection{6/archdischild-2015-308599.322}

We sought to evaluate the use of combined oesophageal multichannel intraluminal impedance and $\mathrm{pH}(\mathrm{MII}-\mathrm{pH})$ monitoring in the Paediatric department of a large tertiary hospital, and to determine whether there is added value of MII-pH monitoring beyond that of conventional $\mathrm{pH}$ monitoring.

We conducted a retrospective study of all paediatric patients who underwent MII-pH monitoring during a two year period $(01 / 01 / 2012-31 / 12 / 2013)$ in a large tertiary hospital. We analysed the demographics, indications for investigation and
MII-pH results for each patient. We assessed the effect of antireflux treatment on MII-pH results and compared the relative contribution of MII data with $\mathrm{pH}$ data in each test.

96 studies were performed on 96 patients (mean age 3 years 8 months, range 2 months to 16 years, 55 male, 41 female). 57 studies produced interpretable results of greater than 20 h' duration. Respiratory symptoms were the most common indication for study. Patients who had taken anti-reflux medication at any point in the week prior to the study were significantly less likely to have an abnormal reflux index (RI; $p<0.05$ ) but were not less likely to have positive symptom correlation. When considering patients off treatment in the week prior to the study, patients with an abnormal RI were found to have a significantly higher frequency of both total reflux and acid reflux events compared to those with a normal RI ( $p<0.001$ ), and were also more likely to have a positive correlation between their symptoms and impedance-detected reflux events $(\mathrm{p}<0.01) .19$ patients had both an interpretable MII-pH study and a contemporaneous oesophageal biopsy; however, no significant correlation was seen between biopsy result and any of the MII-pH indices.

This study provides a comprehensive review of the use of MII-pH monitoring in a large tertiary centre. It reinforces previous literature findings that anti-reflux medication reduces oesophageal acid exposure, but does not necessarily reduce total reflux frequency or symptom correlation. The value added by MII-pH monitoring beyond that of conventional $\mathrm{pH}$ monitoring remains to be established. The results here support the use of RI (derived from $\mathrm{pH}$ monitoring) as an indicator for reflux disease in patients off treatment.

\section{G367(P) A CHUBBY CHILD: IS THIS A SIGN OF HEALTH OR MALNUTRITION?}

F Ajmal, A Copeman. Paediatric Department, New Cross Hospital, The Royal Wolverhampton NHS Trust, Wolverhampton, UK

\subsection{6/archdischild-2015-308599.323}

A fifteen month old British girl presented with severe anaemia, anasarca, dermatitis, hepatomegaly, hypo-pigmented hair, hair loss and global developmental delay. Initial examination revealed the patient to be of appropriate weight however her height was severely stunted. Blood tests revealed severe iron deficiency anaemia, Vitamin B12 deficiency, and various other vitamin/mineral deficiencies, low albumin and total protein levels. A dietary history revealed she was mainly breastfed from a strict vegan mother. A diagnosis of Kwashiorkor was made and management was commenced. There was no evidence of other pathology and safeguarding procedures where implemented to support the family.

This case illustrates a classical diagnosis commonly made in developing countries of the World as a result of malnutrition. It is not commonly encountered in the developed world due to a strong vigilant network of support for all children in the community and financial support for people with lower socioeconomic status in countries such as the UK.

Whilst Kwashiorkor's and other forms of protein-energy malnutrition are commonly encountered in developing countries, clinicians and other healthcare providers training in the West have often not encountered them in clinical practice. This depicts the importance of constant learning and maintaining a broader vision when assessing children. 
G368(P) DYNAMICS OF DEGREE BREACH STRUCTURE OF LIVER AND DEGREE PORTAL HYPERTENSION IN CHILDREN WITH AUTOIMMUNE HEPATITIS AND WILSON'S DESEASE

GV Volynets, NN Evlyukhina, AN Filin, NL Pakhomovskaya. Gastroenterology, Federal State Budgetary Institution (Scientific Centre of Children Health), Moscow, Russian Federation

10.1136/archdischild-2015-308599.324

Aim To determine the degree of disruption of the structure of the liver and the severity of portal hypertension and its dynamics during therapy in patients with autoimmune hepatitis (AIH) and Wilson's disease (WD) in children based on a points system determining the degree of disruption of the structure of the liver.

Methods Analysis of case histories of 176 child at the age from 1 to 17 years (mean age $11.8 \pm 3.5$ years) with WD $(55.4 \%)$ and $\mathrm{AIH}(42.6 \%)$. Of them with cirrhosis $-40.4 \%$, without cirrhosis - 59.6\%. 49 children $(27.8 \%)$ liver transplantation was performed.

Results In children with AIH degree of disruption of the liver structure was $20.4 \pm 9.2 \%$. In children with liver cirrhosis in the outcome of AIH $-24.8 \pm 8.3 \%$, without cirrhosis - $13.2 \pm 5.5 \%$ $(\mathrm{p}<0.001)$. In children with WD degree of disruption of the liver structure at first hospitalisation was $17.7 \pm 12.1 \%(\mathrm{p}=$ $0.305)$. In children with liver cirrhosis in the outcome WD -32.4 $\pm 9.2 \%$, without cirrhosis $-10.5 \pm 4.0 \%$ ( $p<0.001)$. In 49 children, who underwent liver transplantation, the degree of disruption of liver structure was $45.2 \pm 16.2 \%$ ( $p<0.001)$. The degree of disruption of liver structure with AIH after 6 months of therapy $-15.1 \pm 8.0 \%(\mathrm{p}=0.007)$, in children with liver cirrhosis in the outcome of AIH $-16.9 \pm 8.9 \%(p<0.001)$, without cirrhosis $-11.0 \pm 2.3 \%(\mathrm{p}=0.276)$. After 12 months of therapy, the degree of disruption of the structure of the liver in children with AIH $-13.4 \pm 5.2 \%(\mathrm{p}<0.001)$, in children with liver cirrhosis in the outcome of AIH $-15.6 \pm 5.3 \%(\mathrm{p}<0.001)$, in children without cirrhosis was $9.8 \pm 1.8 \%(\mathrm{p}=0.139)$.

Conclusion Scoring system degree of disturbance of the liver structure and severity of portal hypertension can be used as an objective criterion for evaluating the degree of disturbance of liver structure changes in their dynamics on the background of the therapy.

\section{G369(P) PORTAL CAVERNOMA AND THE CHALLENGES FACED IN EARLY INFANCY}

1,2ND Ruth, 'I Van Mourik. 'Hepatology, Birmingham Children's Hospital NHS Foundation Trust, Birmingham, UK; ${ }^{2}$ Medical School, University of Birmingham, Birmingham, UK

\subsection{6/archdischild-2015-308599.325}

We describe the case of an ex 25/40 gestation infant initially referred with failure to thrive to her local hospital. She had a background of tracheo-oesophageal fistula and had a degree of chronic lung disease for which she had home oxygen. She was the first born child to unrelated caucasian parents, and was placed in foster care prior to discharge due to parental drug abuse. Examination at referral centre revealed marked splenomegaly. Ultrasound scan revealed portal cavernoma and she was referred to a tertiary unit for investigation and management.

Prior to review, the patient suffered a significant PR bleed at a corrected gestational age of 4 months. Following transfer she suffered 4 further bleeds, 2 of which resulted in cardiac arrest. This is an unusual presentation in a child so young. She had endoscopy and banding, as well as sclerotherapy on 4 separate ocasions, each time suffering a further bleed 3 days later. She was commenced on anti-hypertensive medications with minimal effect. Octreotide did control the bleeding but each time this was weaned she suffered further bleeding. She went on to have splenorenal shunt surgery which abated the bleeds temporarily prior to eventual splenectomy. She had a prolonged intensive care admission and was eventually discharged home on.

This case report highlights the sequelae of prematurity as well as management of an unusual presentation of portal cavernoma in a patient of this age.

\section{G370(P) FICTITIOUS ILLNESS DUE TO CHRONIC LAXATIVE POISONING: A CASE REPORT}

DC Atukorale, R Jayatunga. Sandwell \& West Birmingham Hospitals NHS Trust, Birmingham, UK

\subsection{6/archdischild-2015-308599.326}

Aims Fictitious Illness is a form of child abuse, where a perpetrator makes a child appear sick by either fabricating symptoms or actually causing harm to the child, in order to gain attention. The child could be poisoned by the perpetrator to induce the symptoms.

Method We report the course of a girl who presented with chronic diarrhoea and severe failure to thrive, as a result of chronic laxative poisoning, by her mother. A review of the English literature (PubMed) revealed only 1 other similar case report in an infant.

Results A girl was admitted repeatedly (11 admissions in 11 months) to hospital between the ages of 3 weeks and 11 months for further evaluation of chronic diarrhoea and severe growth faltering. Her mother reported that she had vomiting, poor feeding and diarrhoea. Her birth weight was $4.07 \mathrm{~kg}\left(91^{\text {st }}\right.$ centile) but at 11 months she was emaciated, weighing only $6.94 \mathrm{~kg}$ (well below the $3^{\text {rd }}$ centile).

She had been extensively investigated for a cause, including regular blood, stool and urine tests, imaging and intestinal biopsies. As the aetiology for her symptoms was not found, she was managed for possible gastro-oesophagial reflux and cow's milk protein intolerance, although neither were proven. But her symptoms continued, despite a multi-disciplinary approach from paediatricians, tertiary gastroenterologists, dieticians, community nursing and health visitor, causing significant malnutrition and delayed development, by 1 year of age.

Incidental finding of Bisacodyl with the child's belongings during an admission led to the suspicion of laxative poisoning. It was confirmed with positive urine toxicology for Bisacodyl on two separate occasions.

Mother was removed from the care of the child with involvement of social services. Following this, she made excellent progress and her weight increased from $6.925 \mathrm{~kg}$ to 9.35 $\mathrm{kg}$ within a period of $31 / 2$ weeks giving an average weight gain of $692 \mathrm{~g}$ per week. In foster care her development returned to normality.

Conclusions We highlight the need to consider this rare diagnosis when extensive investigations fail to identify an underlying cause in infants with severe weight loss due to protracted diarrhoea. 